\title{
Coordinated Risk Mitigation Strategy for Integrated Energy Systems under Cyber-Attacks
}

\author{
Pengfei Zhao, Student Member, IEEE, Chenghong Gu, Member, IEEE and Da Huo
}

\begin{abstract}
The dramatic increase of cyber-attacks on energy systems can cause huge losses, which has drawn extensive attention due to the fast integration of information communication technologies (ICTs). This issue is becoming worse with the integration of electricity and gas systems (IEGS), facilitated by gas generation and new coupling technologies.

This paper investigates the risk and mitigation strategies for IEGS under false data injection attacks (FDIA) in a hierarchical two-stage framework. The FDIA on both electricity and gas systems are modelled through injecting falsified data by adversaries. To mitigate the adverse impacts, a novel two-stage distributionally robust optimization (DRO) is proposed: i) day-ahead operation to determine initial operation scheme and ii) real-time corrective operation with the realization of FDIA and renewable generation uncertainties. A semidefinite programming is formulated for the original problem and it is then solved by a convex optimizationbased algorithm. A typical IEGS is used for demonstration, which shows that the proposed model is effective in mitigating the risks caused by potential FDIA and renewable uncertainties, by optimal coordinating energy infrastructures and load shedding. This work provides system operators with a powerful tool to operate the IEGS with enhanced security against malicious cyber-attacks while accommodating increasing renewable energy. The method can also be easily extended to operating IEGS against other natural attacks.
\end{abstract}

Index Terms-Cyber-attacks, distributionally robust optimization, false data injection attacks, integrated electricity and gas system, real-time operation, risk mitigation.

\section{NOMENCLATURE}

\section{A. Indices and sets}

\section{$t, T \quad$ Index and set for time periods.}

$b, B \quad$ Index and set for electricity buses.

$i_{e}, I_{e} \quad$ Index and set for electricity generators.

$i_{g}, I_{g} \quad$ Index and set for gas wells.

GT Index for gas turbine.

$j, J \quad$ Index and set for renewable generators.

$l_{e}, L_{e} \quad$ Index and set for power lines.

$l_{g}, L_{g} \quad$ Index and set for gas pipelines.

$k_{e}, K_{e} \quad$ Index and set for electric loads.

$k_{g}, K_{g} \quad$ Index and set for gas loads.

\section{B. Parameters}

AIL Attack injection level for FDIA.

Manuscript received October 3, 2019; revised February 13, 2020; accepted April 5, 2020.

P. Zhao, C.Gu (corresponding author) are with the Department of Electronic \& Electrical Engineering, University of Bath, Bath, UK.(email: P. Zhao@bath.ac.uk; C.Gu@bath.ac.uk).

D. Huo is with the School of Engineering, Newcastle University, UK. (email:Da.Huo@newcastle.ac.uk). $\lambda_{i_{e}}^{a}, \lambda_{i_{e}}^{b}, \lambda_{i_{e}}^{c}$

Cost coefficients for of electricity generator $i_{e}$.

$\lambda_{i_{g}} \quad$ Cost coefficient for gas well $i_{g}$.

$\lambda_{i_{e}}^{+}, \lambda_{i_{e}}^{-}$

$\lambda_{i}^{r e}, \lambda_{j}^{r e}$

$\lambda_{k_{e}}^{l s}, \lambda_{k_{g}}^{l s}$

$\omega_{j}^{S}(t)$

$R_{i_{e}}^{+}, R_{i_{e}}^{-}$

$R_{G T}^{+}, R_{G T}^{-}$

$P_{i_{e}, \max }$,

$P_{i_{e}, \min }$

$P_{i_{g}, \max }$,

$P_{i_{g}, \min }$

$P_{G T, \max }$,

$P_{G T, \min }$

$x_{l_{e}}$

$f_{l_{e}, \max }$

$f_{l_{g}, \max }$

$\omega_{j, t}^{s}$

$P r_{l_{g}, \text { max }}$,

$P r_{l_{g}, \text { min }}$

$\gamma_{l_{g}}$

$D_{l_{g}}, L_{l_{g}}$

$F_{l_{g}}$

$R$

$Z$

$\rho_{l_{g}}$

Temp

$P_{k_{e}, t}, P_{k_{g}, t}$

$P_{k_{e}, \max }^{l s}$,

$P_{k_{g}, \max }^{l s}$

$\eta_{i_{e}, t}, \eta_{G T, t}$

Cost coefficient for up and down reserve of electricity generator $i_{e}$.

Regulation cost coefficient for electricity generator $i_{e}$ and renewable generator $j$.

Penalty cost coefficient for electricity and gas load shedding.

Forecasted output of renewable generator $j$ at time $\mathrm{t}$.

Maximum up and down reserve capacity of electricity generator $i_{e}$ at time t.

Maximum up and down reserve capacity of gas turbine GT at time t.

Maximum and minimum output of electricity generator $i_{e}$.

Maximum and minimum output of gas well $i_{g}$.

Maximum and minimum output of gas turbine GT.

Resistance of power line $l_{e}$.

Maximum power flow of line $l_{e}$.

Maximum gas flow of line $l_{g}$.

Forecasted renewable generation at time $t$.

Maximum and minimum gas pressure of gas pipeline $l_{g}$.

Coefficient for Weymouth equation.

Diameter and length of pipeline $l_{g}$.

Pipeline friction coefficient.

Specific gas constant.

Compression factor of pipeline $l_{g}$.

Gas density.

Temperature.

Electricity and gas load at time t.

Maximum electricity and gas load shedding at time $\mathrm{t}$.

Participation factor for reserves of electricity generator and gas turbine at time t. 


\begin{tabular}{|c|c|}
\hline \multicolumn{2}{|c|}{ C. Variables and functions } \\
\hline$P_{i_{e}, t}^{S}, P_{i_{e}, t}^{r e}$ & $\begin{array}{l}\text { Scheduled and regulated output of } \\
\text { electricity generator } i_{e} \text { at time t. }\end{array}$ \\
\hline$P_{i_{g}, t}^{S}, P_{i_{g}, t}^{r e}$ & $\begin{array}{l}\text { Scheduled and regulated output of gas well } \\
i_{g} \text { at time t. }\end{array}$ \\
\hline$P_{G T, t}^{s}, P_{G T, t}^{r e}$ & $\begin{array}{l}\text { Scheduled and regulated output of gas } \\
\text { turbine GT at time t. }\end{array}$ \\
\hline$r_{i_{e}, t}^{+}, r_{i_{e}, t}^{-}$ & $\begin{array}{l}\text { Up and down reserve of electricity } \\
\text { generator } i_{e} \text { at time t. }\end{array}$ \\
\hline$r_{G T, t}^{+}, r_{G T, t}^{-}$ & $\begin{array}{l}\text { Up and down reserve of gas turbine GT at } \\
\text { time t. }\end{array}$ \\
\hline$f_{l_{e}, t}^{s}, f_{l_{e}, t}^{r e}$ & Scheduled and regulated power flow. \\
\hline$f_{l_{g}, t}^{s}, f_{l_{g}, t}^{r e}$ & Scheduled and regulated gas flow. \\
\hline$f_{l_{g}, t}^{i n i}, f_{l_{g}, t}^{t e r}$ & $\begin{array}{l}\text { Gas flow from initial node and to terminal } \\
\text { node of pipeline } l_{g} \text { at time t. }\end{array}$ \\
\hline$P r_{l_{g}, t}^{S}, P r_{l_{g}, t}^{r e}$ & $\begin{array}{l}\text { Scheduled and regulated gas pressure of gas } \\
\text { pipeline } l_{g} \text { at time t. }\end{array}$ \\
\hline $\begin{array}{l}P r_{l g, t}^{s, i n i} \\
\operatorname{Pr}_{l_{g}, t}^{s, t e r}\end{array}$ & $\begin{array}{l}\text { Scheduled gas pressure of initial and } \\
\text { terminal nodes of pipeline } l_{g} \text { at time t. }\end{array}$ \\
\hline $\begin{array}{l}P r_{l_{g}, t}^{r e, i n i} \\
P r_{l_{g}, t}^{r e, t e r}\end{array}$ & $\begin{array}{l}\text { Regulated gas pressure of initial and } \\
\text { terminal nodes of pipeline } l_{g} \text { at time t. }\end{array}$ \\
\hline$P_{k_{e}, t}^{l s}$ & Electricity load shedding at time t. \\
\hline$P_{k_{g}, t}^{l s}$ & Gas load shedding at time $t$. \\
\hline$x, y$ & Vectors of first and second stage variables. \\
\hline$P f()$ & Probability function. \\
\hline$E_{P}[]$ & Expectation over distribution. \\
\hline\langle\rangle & Trace of matrix. \\
\hline$\psi_{0}, \psi_{j}, \Psi_{j k}, \tau$ & Dual variables. \\
\hline D. Uncert & inty \\
\hline$\xi_{j, t}$ & $\begin{array}{l}\text { Real-time renewable power output of } j \text { at } \\
\text { time } t \text {. }\end{array}$ \\
\hline $\begin{array}{l}\Delta P_{k_{e}, t}, \Delta P_{k_{g}, t} \\
\rho_{l_{g}}^{F}\end{array}$ & $\begin{array}{l}\text { FDIA on electricity load, gas load and gas } \\
\text { density at time t. }\end{array}$ \\
\hline$D$ & $\begin{array}{l}\text { Ambiguity set for FDIA and renewable } \\
\text { uncertainty. }\end{array}$ \\
\hline$\mu, \Sigma$ & $\begin{array}{l}\text { Mean vector and covariance matrix for } \\
\text { FDIA and renewable uncertainty. }\end{array}$ \\
\hline$\Theta$ & Second moment matrix. \\
\hline$V S$ & Polyhedral set of extreme points. \\
\hline
\end{tabular}

\section{INTRODUCTION}

$\mathrm{P}$ OWER systems have evolved to be more intelligent, efficient and reliable with the increasing dependence on data communication infrastructures [1]. The information and communication technology (ICT) supports bidirectional information flows and thus enhances the optimal control of the physical power system with better observability and controllability. However, high integration and modernization of ICT can naturally raise threats to power system security [2]. The adversary can launch false data injection attacks (FDIA) to tamper critical data and inject falsified data, which brings serious challenges to state estimators, indirectly affecting system operation and control. In 2015, three Ukrainian regional power distribution companies were attacked by FDIA which caused power outages for 225,000 customers [3].

Existing research of FDIA most focuses on investigating i) maximally launching FDIA to cause damages [4-6], ii) detection algorithm against FDIA [7-8] and iii) mitigation and protection schemes against FDIA [11-12]. As for attack modelling and detection, paper [4] models how an adversary can trigger sequential outages on targeted branches by identifying critical branches. A stochastic model is proposed to design FDIA that affects electricity market by adopting imperfect grid information [5]. Paper [6] proposes an FDIA that can be launched through the approximation on system states based on injection measurements. An online anomaly detection algorithm is used to detect FDIA based on load forecasting and generation scheduling [7], where the minimum attack magnitudes and detection thresholds are determined. A detection and isolation scheme is proposed in [8] by using interval observer based on the physical dynamics of grids.

State estimation is of significance in FDIA detection. However, malicious FDIA can be masked and hidden through judiciously designing residue of bad data detection $[9,10]$. Therefore, mitigation strategy is the final barrier for protecting power systems provided that detection is failed. A corrective scheme is proposed to address overloading and uneconomic dispatch in [11] against the worst-case FDIA. Paper [12] proposes a unit commitment model by using a trilevel optimization model, which is converted into a bilevel mixed integer programming problem.

The increasing demand growth of both power and gas systems, low price of natural gas resources, and conversion technologies between the two systems, e.g., gas-fired units and power-to-gas facilities, have promoted the interdependency between power and gas systems. Consequently, modelling and optimizing the two independent systems as an entity can facilitate the economy and security for both systems. Integrated electricity and gas systems (IEGS) realizes the coordination between energy infrastructures in both power and gas systems.

The electricity and natural gas systems are increasingly independent, interconnected by many coupling technologies to form IEGS. Conversion technologies between the two systems include gas-fired units and power-to-gas facilities. Combined heat and power (CHP) enables the conversion of gas to both heat and electricity [13, 14]. Power-to-gas (P2G) facilities can convert excessive renewable energy to synthetic natural gas [ $[15$, 16]. The conversion from gas to power is mainly realized by utilizing gas turbines $[17,18]$. In some compressor stations of natural gas systems, electricity is used to drive compressors. The interdependency produces many benefits, including enhanced security of supply, more absorption of renewable energy, but there are also many adverse impacts. Cascading failures in one system can propagate to the other and the cyberattacks on one system can affect the security of the other.

Existing IEGS literatures on making use of its unique interdependency generally concentrates on i) operation under 
normal conditions, ii) security-based operation under reliability issues and iii) resilience enhancement and operation strategies under natural disasters [19-23]. Paper [19] proposes an optimal operation scheme for IEGS considering electricity demand response and the impact on the entire system is profound due to the strong interdependency of power and gas systems. A-low carbon IEGS community with heat delivery system is proposed in [20], where the uncertainties of renewable generation and demand are handled by stochastic optimization (SO). Paper [21] models a security-constrained unit commitment against $N-k$ outages by using robust optimization (RO). Nonlinear gas flow is relaxed into a second-order cone problem for convexity. To enhance IEGS resilience, a robust network hardening strategy is proposed in [22], considering natural disaster uncertainties. Paper [23] proposes a minimax-regret robust unit commitment model for enhancing the resilience of IEGS against the extreme weather obtained by spatial dynamic method.

Leveraging between $\mathrm{RO}$ and SO, distributionally robust optimization (DRO) is widely applied in power systems to handle uncertainties [24-28]. SO either assumes specific knowledge of probability distributions or requires a large number of uncertainty samples, which is not always practical and can cause high computational burden. RO accommodates uncertainties in predefined uncertainty sets and considers the worst-case scenario, which could have extremely low probability and thus produces over-conservative results. DRO, taking the advantage of distributional information, e.g., moment information, deals with uncertainties within a feasible set, called ambiguity set. Therefore, compared with RO, DRO determines expected results over all possible distributions, which are less-conservative. Compared with SO, DRO avoids intensive computation, thus improving calculation efficiency. Paper [26] applies DRO to a risk-based optimal gas-power flow. An optimal scheduling of IEGS considering electricity and gas load uncertainties is proposed in [27].

Due to the strong interdependency between electricity and gas systems in IEGS, the FDIA on either electricity or gas system can propagate to each other. The adverse impact can be exaggerated when there is high uncertain renewables in the electricity system. But limited effort is dedicated to studying the impact of FDIA on IEGS. This paper proposes a two-stage risk mitigation strategy to address the uneconomic operation of IEGS under FDIA considering renewable generation uncertainties. FDIA is assumed to attack both electricity and gas meter readings, including i) load measurement of electricity and gas systems and ii) gas density measurement. In the first stage, the day-ahead optimization determines an optimal IEGS scheduling scheme based on forecasted renewable generation without considering potential FDIA. In the second stage, both FDIA and renewable uncertainties are revealed, a real-time corrective optimization is built to minimize the attack impacts through load shedding and adjusting generation output. The original problem is converted into equivalent semidefinite programming (SDP) and a constraint generation algorithm (CGA) is adopted to solve the SDP problem. For conciseness and simplicity, the proposed distributionally robust FDIA mitigation scheme is represented by DR-FMS.
The major contribution of this paper is as follows:

1) It models FDIA in an IEGS for the first time, particularly on natural gas load and density measurement, where existing research only focuses on FDIA on electricity systems.

2) Uncertainties of renewable resources are considered in the proposed model as they can worsen system operation conditions during FDIA, compared to existing FDIA papers $[11,12]$ that ignore the impact of renewable uncertainties.

3) Compared to $\mathrm{SO}$ and $\mathrm{RO}$ for modelling FDIA, the ambiguous distribution of DRO developed in this paper, which is less data-dependent and conservative, can better characterize uncertain variables.

4) A two-stage FDIA mitigation scheme is proposed for the first time to conduct the day-ahead and real-time operation, which is more powerful and convenient to be used by system operators to ensure the efficiency and security of the IEGS.

The rest of this paper is organized as follows. Section II models FDIA for integrated systems.Section III presents problem formulation of the DR-FMS. The DRO methodology regarding and associated reformulations are given in Section IV. Section V demonstrates case studies and performance of the DR-FMS. Finally, the conclusion is given in Section VI.

\section{ATTACK MODELLING}

This section presents the attack modelling for electricity and gas system. State estimation is a powerful tool to detect FDIA by processing raw data measurements, but a successful FDIA can be undetectable by adversary's stealthy design.

\section{A. Attacks on Electricity System}

The nonlinear relationship between state variable $x$ and measurement $z$ is given in (1), where $h(x)$ denotes the nonlinear vector function of $x$ and $e$ is the error measurement. Based on DC state estimation, equation (1) can be transformed into (2), where $H$ represents the Jacobian matrix.

$$
\begin{gathered}
z=h(x)+e \\
z=H x+e
\end{gathered}
$$

After the realization of FDIA, the measurement vector $z$ becomes $z_{b a d}=z+a$, and the estimated state vector can be represented as $\hat{x}_{b a d}=\hat{x}+c$ where $a$ is attack vector and $c$ is the resulted deviation vector of state variable after FDIA. Accordingly, to determine the estimated state variable, $\hat{x}_{\text {bad }}$ can be formulated as:

$$
\hat{x}_{\text {bad }}=\left(H^{\prime} W H\right)^{-1} H^{\prime} W z_{b a d}
$$

The largest normalized residual (LNR) can be used to detect and identify measurement errors by (4). If the residual is less than a threshold $\varepsilon$, then the state estimate is valid without FDIA.

$$
L N R=\|z-H \hat{x}\| \leq \varepsilon
$$

Then, equation (5) representing LNR is given based on (3) and (4). Finally, equation (6) is obtained.

$$
\begin{gathered}
L N R=\left\|z+a-H\left(\left(H^{\prime} W H\right)^{-1} H^{\prime} W z_{b a d}\right)\right\| \\
L N R=\|z-H \hat{x}+(a-H c)\|
\end{gathered}
$$

If $a$ is the linear combination of $H$ and $c$, i.e., $a=H c$, then $L N R=\|z-H \hat{x}\|$ has no change of residual. Therefore, a successful FDIA is launched which can evade detection. 
TABLE I

TWO-STAGE MITIGATION FRAMEWORK

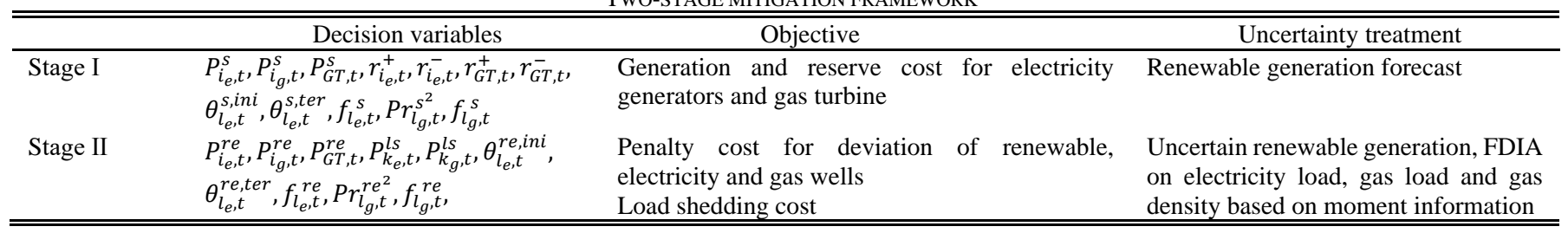

As a special case of FDIA, load measurement can be attacked according to $[9,10]$ by enforcing the sum of load attack vector to be zero in (7). For simplicity, the FDIA on electricity load is represented by EL-FDIA. Equation (8) constraints the attack deviation by attack injection level (AIL).

$$
\begin{gathered}
\sum \Delta P_{k_{e}, t}=0 \\
-A I L_{k_{e}} P_{k_{e}, t} \leq \Delta P_{k_{e}, t} \leq A I L_{k_{e}} P_{k_{e}, t}
\end{gathered}
$$

\section{B. Attack on Gas System}

The FDIA on a gas system is considered on both gas load and density measurement. Similar to FDIA on electricity load measurement, the FDIA on gas load measurement and density are given in (9) and (10), namely GL-FDIA and GD-FDIA. The changed gas density measurement results in a change of Weymouth coefficient $\gamma_{l_{g}}$. Accordingly, the initial coefficient in (11) is changed to (12) under FDIA.

$$
\begin{gathered}
0 \leq \Delta P_{k_{g}, t} \leq A I L_{k_{g}} P_{k_{g}, t} \\
\rho_{l_{g}} \leq \rho_{l_{g}}^{F} \leq\left(1+\beta_{d}\right) \rho_{l_{g}} \\
\gamma_{l_{g}}=\left(\frac{\pi}{4}\right)^{2} \frac{\alpha^{2} D_{l_{g}}^{5}}{L_{l_{g}} F_{l_{g}} R Z \rho_{l_{g}}{ }^{2} \text { Temp }} \\
\gamma_{l_{g}}^{F}=\left(\frac{\pi}{4}\right)^{2} \frac{\alpha^{2} D_{l_{g}}^{5}}{L_{l_{g}} F_{l_{g}} R Z \rho_{l_{g}}^{F}{ }^{2} \text { Temp }}
\end{gathered}
$$

\section{TWO-STAGE RISK MITIGATION SCHEME}

The risk mitigation for IEGS under potential FDIA consists of: i) day-ahead operation without considering FDIA or renewable uncertainties and ii) real-time operation actions for a corrective mitigation scheme under potential FDIA with the realization of renewable uncertainties. The objective functions and associated constraints are presented in this section.

\section{A. DR-FMS Objective Function}

A summary of decision variables, objective functions and uncertainty modelled in the two stages is presented in TABLE I. Equation (13) presents the day-ahead operation objective function in the first stage. The first four terms represent the generation cost of electricity and gas respectively. Reserve costs of electricity generators are shown in the rest. It is noted that the reserve capacity is prepared for FDIA and uncertainties from renewable resources.

$$
\begin{gathered}
\Gamma_{1}=\min \sum_{i_{e} \in I_{e}, i_{g} \in I_{g}, t \in T} \lambda_{i_{e}}^{a} P_{i_{e}, t}^{s}{ }^{2}+\lambda_{i_{e}}^{b} P_{i_{e}, t}^{s}+\lambda_{i_{e}}^{c}+\lambda_{i_{g}} P_{i_{g}, t}^{s} \\
+\lambda_{i_{e}}^{+} r_{i_{e}, t}^{+}+\lambda_{i_{e}}^{-} r_{i_{e}, t}^{-}
\end{gathered}
$$

The real-time objective function in the second stage is given in (14), which mitigates the impact against the presence of FDIA and renewable uncertainty. The first three terms represent the penalty cost for renewable generators, electricity generators and gas wells when regulated generation deviates from scheduled generation. The final two terms represent electricity and gas load shedding cost.

$$
\begin{aligned}
\Gamma_{2}=\min \sum_{i_{e} \in I_{e}, i_{g} \in I_{g}, t \in T, k_{e} \in K_{e}, k_{g} \in K_{g}}+\lambda_{j}^{r e}\left|\omega_{j, t}^{s}-\xi_{j, t}\right| \\
+\lambda_{i_{e}}^{r e}\left|P_{i_{e}, t}^{s}-P_{i_{e}, t}^{r e}\right|+\lambda_{i_{g}}^{r e}\left|P_{i_{g}, t}^{s}-P_{i_{g}, t}^{r e}\right| \\
+\lambda_{k_{e}}^{l s} P_{k_{e}, t}^{l s}+\lambda_{k_{g}}^{l s} P_{k_{g}, t}^{l s}
\end{aligned}
$$

\section{B. Proposed Coordinated Modelling of IEGS}

The IEGS is a tight coupling entity due to the strong interdependency between electricity and gas systems. Accordingly, the two systems should be modelled together by one decision maker. The modelling of IEGS in the existing literatures can be generally categorized into three types: i) Modelling from the perspective of electricity system operators, which overlooks the operational and security constraints of gas system [29, 30]. This ignorance will cause the physical gas flow violation due to the renewable power fluctuation and load variability; ii) Sequential optimization for IEGS [31, 32], which firstly solves the power system model for determining the optimal schedule for generators while neglects the operational constraints of gas system. Based on the obtained solution from power system, the gas system can be solved; and iii) Cooptimization for IEGS which optimizes the comprehensive objective simultaneously [13-17].

This paper provides a simultaneous coordinated model for the electricity and gas systems. Due to the different characteristics of electricity and gas systems, the operational constraints of two systems are nonrelevant. However, the two systems are solved interdependently with the gas turbine interconnected between the two systems. The gas flow through the gas turbine can be used to generate power flow, which is considered as the supplement for electricity system.

\section{Day-ahead Operation}

The day-ahead operation is implemented based on renewable generation forecast without FDIA risks, whose constraints are in (15)-(28). Constraint (15) and (16) limit the reserve capacity for electricity generators and gas turbine. The scheduled output of electricity generators and gas turbine are enforced within limits in (17) and (18). The linearized DC power flow is given in (19) and (20). Constraint (21) ensures the power balance. Gas well output is limited in (22). Gas pressure is limited in (23). Constraint (24) means the pressure at the initial node is larger than the terminal node since the proposed gas system has a radial topology. Weymouth gas equation for describing gas flow is shown in (25) and (26), where the coefficient is defined in (11). Gas turbine connects two interdependent systems as a coupled infrastructure. Constraint (27) presents the transformation from gas flow injection to power generation. The gas balancing condition is given in (28). 


$$
\begin{aligned}
& 0 \leq r_{\{\cdot\}, t}^{+} \leq R_{\{\cdot\}}^{+},\{\cdot\}=i_{e}, G T \\
& 0 \leq r_{\{\cdot\}, t}^{-} \leq R_{\{\cdot\}}^{-},\{\cdot\}=i_{e}, G T \\
& P_{\{\cdot\}, t}^{s}+r_{\{\cdot\}, t}^{+} \leq P_{\{\cdot\}, \max },\{\cdot\}=i_{e}, G T \\
& P_{\{\cdot, \min } \leq P_{\{\cdot\}, t}^{S}-r_{\{\cdot\}, t}^{-},\{\cdot\}=i_{e}, G T \\
& x_{l} f_{l_{e}, t}^{s}=\left(\theta_{l_{e}, t}^{s, i n i}-\theta_{l_{e}, t}^{s, t e r}\right) \\
& -f_{l_{e}, \max }^{s} \leq f_{l_{e}, t}^{s} \leq f_{l_{e}, \max }^{s} \\
& \sum_{i_{e} \in I_{e}} P_{i_{e}, t}^{s}+P_{G T, t}^{s}+\sum_{j \in J} \omega_{j, t}^{s}+\sum_{l_{e} \in L_{e}} f_{l_{e}, t}^{s, i n i}-\sum_{l_{e} \in L_{e}} f_{l_{e}, t}^{s, t e r} \\
& =\sum_{k_{e} \in K_{e}} P_{k_{e}, t} \\
& P_{i_{g}, \min } \leq P_{i_{g}, t}^{S} \leq P_{i_{g}, \max } \\
& P r_{l_{g}, \text { min }}^{2} \leq P r_{l_{g}, t}^{s^{2}} \leq P r_{l_{g}, \max }^{2} \\
& P r_{l_{g}, t}^{s, i n i} \geq P r_{l_{g}, t}^{s, t e r} \\
& f_{l_{g}, t}^{s{ }^{2}}=\gamma_{l_{g}}\left(P r_{l_{g}, t}^{s, i n i^{2}}-P r_{l_{g}, t}^{s, t e r^{2}}\right) \\
& 0 \leq f_{l_{g}, t}^{s} \leq f_{l_{g}, \max }^{s} \\
& P_{G T, t}^{s}=c_{G T} f_{l_{g}, G T}^{s} \\
& \sum_{i_{g} \in I_{g}} P_{i_{g}, t}^{s}+\sum_{l_{g} \in L_{g}} f_{l_{g}, t}^{s, i n i}-\sum_{l_{g} \in L_{g}} f_{l_{g}, t}^{s, t e r}=\sum_{k_{g} \in K_{g}} P_{k_{g}, t}
\end{aligned}
$$

\section{Real-time Risk Mitigation}

Considering potential FDIA and uncertainties of renewable resources, real-time risk mitigation is presented in the second stage to mitigate uneconomic dispatch. The approach is distributionally robust against FDIA and renewable uncertainty. The regulated generator and gas turbine output are shown in (29). Constraint (30) represents the electricity and gas load shedding limits. The limits considered in the model is based on the existing research [33]. The regulated power flow is constrained in (31) and (32). Constraint (33) presents the limits for gas wells. The regulated gas pressure and flow are limited in (34)-(37). Based on (12), the new Weymouth coefficient $\gamma_{l_{g}}^{F}$ influenced by the attacked gas density is applied. Constraint (38)-(40) show that the power imbalance caused by renewable uncertainties should be offset by adjusting the reserves of generators and gas turbine. Specifically, constraint (38) ensures the deviation of renewable generation is within the range of up and down reserve limits. In (39) and (40), the adjustment factor $\eta_{i_{e}, t}$ and $\eta_{i_{g}, t}$ are the regulation commitment from generators and gas turbine to mitigate renewable uncertainties. Constraint (41) presents the regulated power generation of gas turbine. Constraint (42) and (43) ensure power and gas balance in the second stage.

$$
\begin{gathered}
P_{\{\cdot\}, t}^{r e}-r_{\{\cdot\}, t}^{-} \leq P_{\{\cdot\}, t}^{r e} \leq P_{\{\cdot\}, t}^{r e}+r_{\{\cdot\}, t}^{+},\{\cdot\}=i_{e}, G T \\
0 \leq P_{\{\}, t \leq}^{l s} \leq P_{\{\cdot\}, \max }^{l s},\{\cdot\}=k_{e}, k_{g} \\
x_{l} f_{l_{e}, t}^{r e}=\left(\theta_{l_{e}, t}^{r e, i n i}-\theta_{l_{e}, t}^{r e, t e r}\right) \\
-f_{l_{e}, \text { max }}^{r e} \leq f_{l_{e}, t}^{r e} \leq f_{l_{e}, \text { max }}^{r e} \\
P_{i_{g}, \min } \leq P_{i_{g}, t}^{r e} \leq P_{i_{g}, \max } \\
\operatorname{Pr}_{l_{g}, \min }^{2} \leq P r_{l_{g}, t}^{r e} \leq P r_{l_{g}, \max }^{2} \\
\operatorname{Pr}_{l_{g}, t}^{r e, i n i} \geq P r_{l_{g}, t}^{r e, t e r}
\end{gathered}
$$

$$
\begin{gathered}
f_{l_{g}, t}^{r e 2}=\gamma_{l_{g}}^{F}\left(P_{l_{g}, t}^{r e, i n i^{2}}-P_{l_{g}, t}^{r e, t e r^{2}}\right) \\
0 \leq f_{l_{g}, t}^{r e} \leq f_{l_{g}, \max } \\
r_{\{\cdot, t}^{-} \leq \eta_{\{\cdot\}, t} \sum_{j \in J}\left(\omega_{j, t}^{s}-\xi_{j, t}\right) \leq r_{\{\cdot\}, t}^{+},\{\cdot\}=i_{e}, G T \\
0 \leq \eta_{\{\}, t} \leq 1,\{\cdot\}=i_{e}, G T \\
\sum_{i_{e} \in I_{e}} \eta_{i_{e}, t}+\sum_{i_{g} \in I_{g}} \eta_{g_{g}, t}^{G T}=1 \\
P_{G T, t}^{r e}=c_{G T} f_{l_{g}, G T}^{r e} \\
\sum_{i_{e} \in I_{e}} P_{i_{e}, t}^{r e}+\sum_{j \in J} \xi_{j, t}+P_{G T, t}^{r e} \\
=\sum_{k_{e} \in K_{e}} P_{k_{e}, t}+\Delta P_{k_{e}, t}-P_{k_{e}, t}^{l s} \\
\sum_{i_{g} \in I_{g}} P_{i_{g}, t}^{r e}+\sum_{l_{g} \in L_{g}} f_{l_{g}, t}^{r e, i n i}-\sum_{l_{g} \in L_{g}} f_{l_{g}, t}^{r e, t e r} \\
=\sum_{k_{g} \in K_{g}} P_{k_{g}, t}+\Delta P_{k_{g}, t}-P_{k_{g}, t}^{l s}
\end{gathered}
$$

Constraint (25) contains one nonlinear term, i.e., ' $f_{l_{g, t}}^{s}$, ' and constraint (36) contains two nonlinear terms, i.e., ' $f_{g, t}^{r e 2 \text {, and }}$ ' $\gamma_{l_{g}}^{F}\left(P r_{l_{g}, t}^{i n i^{2}}-P r_{l_{g}, t}^{t e r^{2}}\right)$ '. They need to be linearized for obtaining convex functions and guaranteeing global optimal solutions. A sufficiently large constant $M_{l g}$ can be used to linearize ' $\gamma_{l_{g}}^{F}\left(P r_{l_{g}, t}^{i n i^{2}}-P r_{l_{g}, t}^{t e r^{2}}\right)$ ', shown in (44) and (45). The bilinear term ' $f_{l g, t}^{s}$ ', can be linearized by piecewise linear approximation by separating nonlinear function into pieces. Readers are referred to [34] for details. It should be noted that ' $P r_{l_{g}, t}^{i n i^{2}}$ ' does not require linearization since it is regarded as squared form throughout the paper.

$$
\begin{aligned}
& f_{l_{g}, t}^{r e 2} \leq\left(P r_{l_{g}, t}^{r e, i n i^{2}}-P r_{l_{g}, t}^{r e, t e r^{2}}\right)+\left(1-\gamma_{l_{g}}^{F}\right) M_{l_{g}} \\
& f_{l_{g}, t}^{r e e^{2}} \geq\left(P r_{l_{g}, t}^{r e, i n i^{2}}-P r_{l_{g}, t}^{r e, t e r^{2}}\right)+\left(1-\gamma_{l_{g}}^{F}\right) M_{l_{g}}
\end{aligned}
$$

\section{METHODOLOGY}

The two-stage DR-FMS is a minmax DRO problem, which is solved by the SDP based model. Firstly, the linear DRO problem is represented by a compact matrix form. Then, the family of possible uncertainty distributions for FDIA and renewable uncertainties is defined by an ambiguity set. Finally, the dual problem of DR-FMS is formulated, solved by CGA.

\section{A. Formulation in Brevity}

Matrices and vectors are used to represent the original problem for notation abbreviation. The objective function (46) represents (13) and (14) to minimize the sum of the first-stage objective $c^{\prime} x$ and the expected second-stage objective $E_{P f}[Q(x, \xi)]$. The random parameter $\xi$ is used to represent both $\Delta P_{k}$ and $\xi_{j}$, which is sampled from a family of distributions $P f$.

$$
\begin{gathered}
\min _{x \in X} c^{\prime} x+\sup _{P f \in D \xi, \xi=\Delta P_{k}, \xi_{j}} E_{P f}[Q(x, \xi)] \\
\text { s.t. } A x \leq b, \\
Q(x, \xi)=\min _{y} f^{\prime} y
\end{gathered}
$$




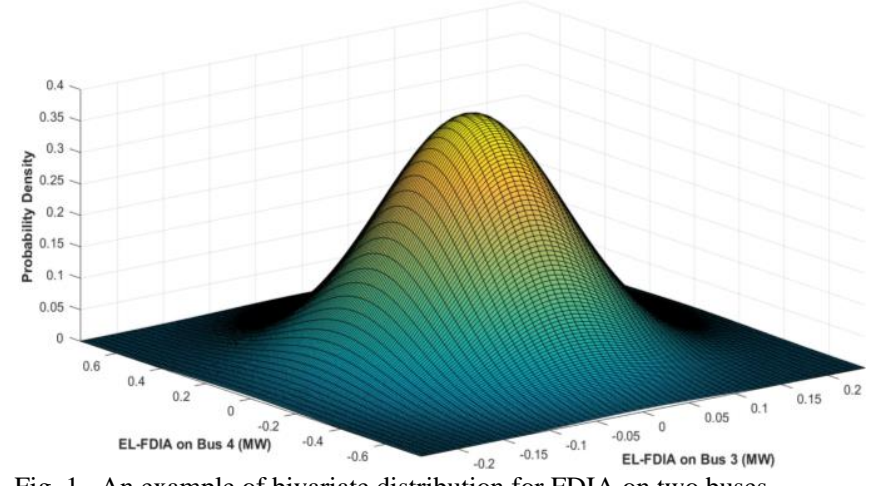

Fig. 1. An example of bivariate distribution for FDIA on two buses.

$$
\text { s.t. } E x+F y+G \xi \leq h \text {, }
$$

The first-stage constraints are shown in (47). Equation (48) and (49) represent the recourse function, where vector $f$ denotes the coefficient of (14).

\section{B. Ambiguity Set for DR-FMS}

The uncertainties of FDIA and renewable power generation can be captured by ambiguity sets that define a family of distributions. Based on limited historical data, moment information, i.e., mean and covariance are obtained to construc empirical point-estimates. The proposed ambiguity set is given in (50) to characterize both uncertain FDIA and renewable generation, which guarantees i) the integral of distribution of $\xi$ is 1 , and ii) the second moments are known.

$$
D=\left\{f(\xi) \mid \begin{array}{c}
\mathrm{P}\{\xi\}=1 \\
\mathrm{E}\{\xi\}=\mu \\
\mathrm{E}\left\{\xi(\xi)^{\prime}\right\}=\Sigma+\mu(\mu)^{\prime}
\end{array}\right\}
$$

The ambiguity set used to characterize uncertain variables is composed of mean and covariance information. Intuitively, a certain set of mean vector and covariance matrix contains all possible probability distributions. Fig. 1 depicts the bivariate distribution for EL-FDIA on two buses with a fixed mean and covariance information. In this case, DRO searches for the distribution that cause the worst-case solution. Fig. 1 only shows one possible distribution, but other distributions can have different shape due to the ignorance of higher order moment information, e.g., kurtosis and skewness.

\section{Equivalent Dual Form}

To obtain a 'min' form of the second-stage problem, dual reformulation is required for the inner problem ' $\mathrm{min}$ sup $E_{P f}[Q(x, \xi)]$ '. The second-stage problem $E_{P f}[Q(x, \xi)]$ is an infinite-dimensional linear problem and the primal form is given in (51)-(55).

$$
\begin{gathered}
S(x)^{\text {primal }}=\max _{P f \in D \xi} \int_{\Xi} Q(x, \xi) P f(\xi) d \xi \\
\text { s.t. } P f(\xi) \geq 0, \forall \xi \in \Xi \\
\int_{\Xi} P f(\xi) d \xi=1 \\
\int_{\Xi} \xi^{m} P f(\xi) d \xi=\mu_{m}, m=1,2, \ldots, \Xi \\
\int_{\Xi} \xi^{m} \xi^{n} P f(\xi) d \xi=\Sigma_{m n}+\mu_{m} \mu_{n}, m, n=1,2, \ldots, \Xi
\end{gathered}
$$

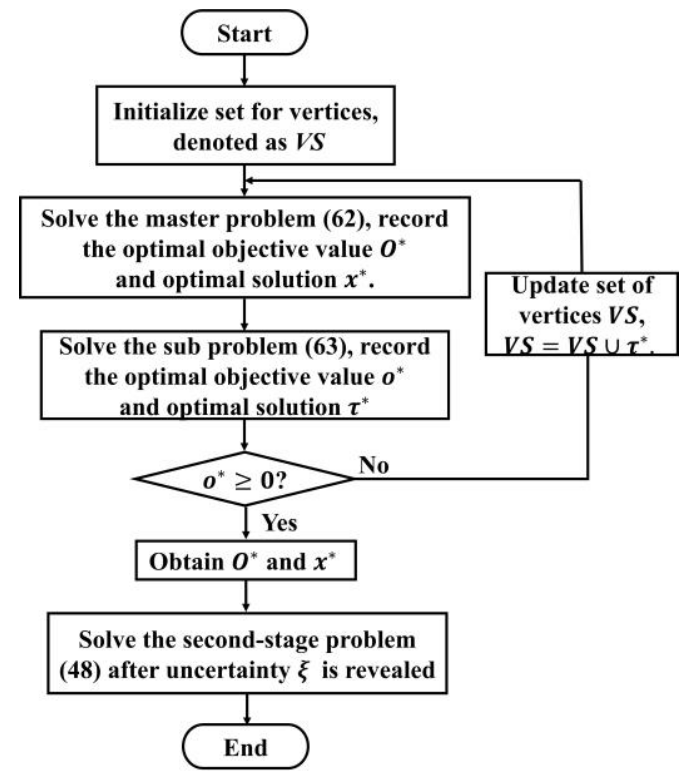

Fig. 2. Flowchart of constrained generation algorithm.

For tractability, the primal form needs to be recast as (56)(57), where $\psi_{0}, \psi_{j}$ and $\Psi_{j k}$ are dual variables associated with constraints (29) to (43), and $\Theta$ represents $\Sigma+\mu(\mu)^{\prime}$. When the weak duality holds, $S(x)^{\text {primal }} \leq S(x)^{\text {dual }}$. However, (55) ensures that the strong duality holds when $\Theta$ is strictly positive definite and thus $S(x)^{\text {primal }}=S(x)^{\text {dual }}$ [35]. Accordingly, now the problem with an infinite number of variables is transformed into one with a finite number of variables (56)-(57), which is easier to solve.

$$
\begin{gathered}
S(x)^{\text {dual }}=\min _{\Psi, \psi, \psi_{0}}\left\langle\Psi^{\prime} \Theta\right\rangle+\psi^{\prime} \mu+\psi_{0} \\
\text { s.t. }(\xi)^{\prime} \Psi \xi+\psi^{\prime} \xi+\psi_{0} \geq Q(x, \xi) \\
\forall \xi \in \Xi
\end{gathered}
$$

The new compact form of DR-FMS is:

$$
\min _{x \in X} c^{\prime} x+S(x)^{d u a l}
$$

\section{SDP Reformulation}

Problem (58) is a semi-infinite-dimensional program which contains an infinite number of constraints. Thus, it is required to be transformed into a closed form [36]. By introducing the new dual variable $\tau$, a positive quadratic function in (59) can be obtained from (48). VS denotes the polyhedral set of extreme points and $N_{v}$ is the set of vertices of feasible region in $V S$.

$$
\begin{gathered}
\max _{u \in V S} \tau^{\prime}(b-E x-G \xi) \\
V S=\left\{\tau \mid F^{\prime} \tau=f, \tau \leq 0\right\} \\
(\xi)^{\prime} \Psi \xi+\left(\psi+G^{\prime} \tau^{i}\right)^{\prime} \xi+\psi_{0}-(h-E x) \tau^{i} \geq 0 \\
\forall \xi \in \Xi, i=1,2, \ldots, N_{v}
\end{gathered}
$$

In summary, the SDP form of DR-FMS is as follow, which is the master problem.

$$
\begin{gathered}
\min _{x, \Psi, \psi, \psi_{0}} c^{\prime} x+\left\langle\Psi^{\prime} \Theta\right\rangle+\psi^{\prime} \mu+\psi_{0} \\
{\left[\begin{array}{l}
\xi \\
1
\end{array}\right]^{\prime}\left[\begin{array}{cc}
\Psi & \frac{1}{2}\left(\psi+G^{\prime} \tau^{i}\right) \\
\frac{1}{2}\left(\psi+G^{\prime} \tau^{i}\right)^{\prime} & \psi_{0}-(h-E x)^{\prime} \tau^{i}
\end{array}\right]\left[\begin{array}{l}
\xi \\
1
\end{array}\right] \geq 0} \\
\forall \xi \in \Xi, i=1,2, \ldots, N_{v}, x \in X, \forall \tau^{i} \in V S
\end{gathered}
$$




\section{E. Constraint Generation Algorithm}

A large number of constraints with infinite cardinality of $V S$ cause high computational burden. CGA initially enumerates a subset of vertices and incorporates more vertices step by step. This relaxation method can efficiently solve the proposed problem, which is separated into a master and sub problem in (62) and (63). The flowchart of the CGA is given in Fig. 2.

$$
\begin{gathered}
\left(\xi_{s}\right)^{\prime} \Psi \xi_{s}+\psi^{\prime} \xi_{s}+\psi_{0}-\left(h-E x-G \xi_{s}\right)^{\prime} \tau \geq 0 \\
\text { s.t. } \forall \xi \in \Xi, \tau \in V S
\end{gathered}
$$

\section{CASE STUdies}

A combined IEEE 30-bus electricity system and a 6-node gas system is used to test the effectiveness of the DR-FMS through the extensive case studies. In the case studies, three types of FDIA are considered, namely EL-FDIA, GL-FDIA and GDFDIA, which represent FDIA on electricity load, gas load and gas density, respectively. For EL-FDIA, the total load is unchanged, which is the fundamental condition of FDIA for evading the detection. The modelling of EL-FDIA is discussed in section II-A, which can be also found in [9, 11, 37]. The following 10 cases are considered:

Case 1: Single-stage deterministic optimization for IEGS without considering FDIA or renewable uncertainty.

Case 2: RO based FMS with three types of FDIA $(A I L=5 \%)$.

Case 3: Case 2 considering renewable uncertainty $(A I L=5 \%)$.

Case 4: Two-stage DR-FMS considering FDIA on electricity $\operatorname{load}(A I L=5 \%)$.

Case 5: Case 4 considering FDIA on both gas and electricity $\operatorname{load}(A I L=5 \%)$.

Case 6: Case 5 considering FDIA on gas density $(A I L=5 \%)$.

Case 7: Case 6 considering renewable uncertainty $(A I L=5 \%)$.

Case 8-10: Case 7 with $A I L=10 \%, 15 \%$ and $20 \%$.

In case 3 and 7 , the addition of renewable uncertainty is considered and the AIL is still $5 \%$ as case 2 and 4 . The proposed test network is shown in Fig. 3, which contains 30 buses, 6 electricity generators, 2 renewable generators, 2 gas wells, 21 electricity loads and 3 gas loads. The renewable generators are connected to bus 22 and 25 with 60MW for each output. Parameters of electricity generators, gas wells are given in TABLEs II and III, which can be found in [38].

The method for case 1 is a deterministic global optimization for solving linear programming. The reasons for not using metaheuristic optimization methods are: i) The deterministic linear programming problem solved by deterministic global optimization and metaheuristic optimization methods have similar results [39-41]; ii) The focus of this paper is to address FDIA and design mitigation schemes. The deterministic optimization method in case 1 is only used for comparison; iii) In practice, system operators implement economic dispatch after the data-filtering by state estimators, which requires high computational efficiency;; and iv) Metaheuristic methods, such as genetic algorithm and particle swarm optimization, easily converge prematurely and could be trapped into a local minimum, particularly with complex problems [42].

It should be noted that the DR-FMS considers the worst-case uncertainty distribution for both FDIA and renewable energy from all candidate distributions. Based on the partial distributional information, i.e., mean value vector and covariance matrix, DR-FMS can test all possible distributions
TABLE II

\begin{tabular}{cccc}
\multicolumn{4}{c}{ PARAMETERS OF GAS WELLS } \\
\hline \hline \multirow{2}{*}{ Node No. } & $\begin{array}{c}P_{i_{g}, \max } \\
(\mathrm{kcf} / \mathrm{h})\end{array}$ & $\begin{array}{c}P_{i_{g}, \min } \\
(\mathrm{kcf} / \mathrm{h})\end{array}$ & $\lambda_{i_{g}}$ \\
\hline 4 & 35 & 10 & 2.2 \\
6 & 70 & 20 & 2 \\
\hline \hline
\end{tabular}

TABLE III

\begin{tabular}{|c|c|c|c|c|c|c|}
\hline $\begin{array}{l}\text { Bus } \\
\text { No. }\end{array}$ & $\begin{array}{l}P_{i_{e}, \min } \\
\text { (MW) }\end{array}$ & $\begin{array}{l}P_{i_{e}, \max } \\
(\mathrm{MW})\end{array}$ & $\begin{array}{r}R_{i_{e}}^{+}, R_{i_{e}}^{-} \\
(\mathrm{MW})\end{array}$ & $a_{i_{e}}$ & $b_{i_{e}}$ & $c_{i_{e}}$ \\
\hline 1 & 50 & 200 & 20 & 0.004 & 2 & 6 \\
\hline 2 & 20 & 80 & 16 & 0.002 & 2 & 6 \\
\hline 5 & 15 & 50 & 10 & 0.006 & 1 & 8 \\
\hline 8 & 10 & 35 & 7 & 0.008 & 3 & 10 \\
\hline 11 & 10 & 30 & 10 & 0.025 & 3 & 18 \\
\hline 13 & 12 & 40 & 16 & 0.025 & 3 & 18 \\
\hline
\end{tabular}

GENERATOR PARAMETERS
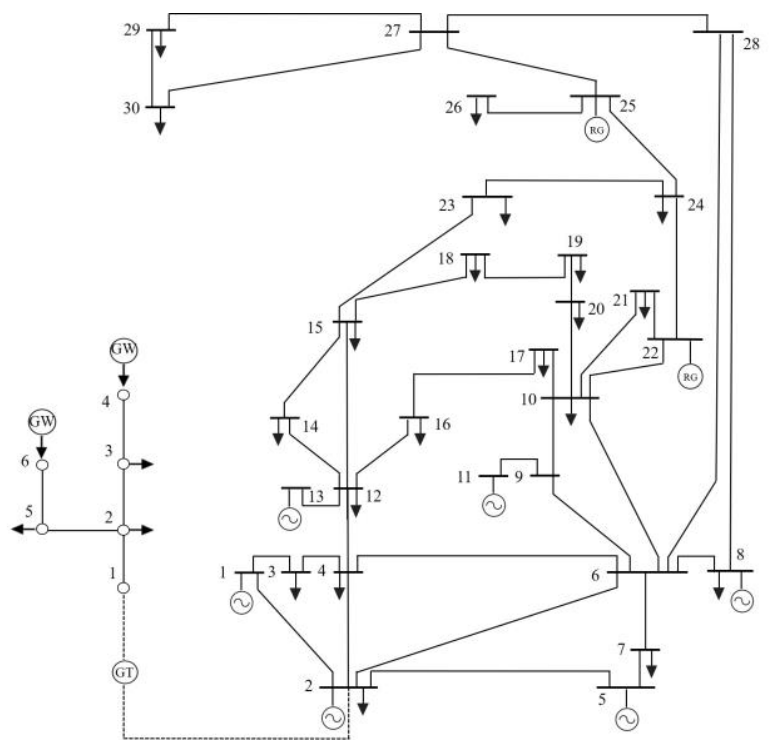

Fig. 3. Modified IEEE 30-bus system.

modelled by moment information. Accordingly, this worstdistribution oriented mitigation scheme is a data-driven approach and actually tests a variety of scenarios.

\section{A. Studies on Economic Performance}

Firstly, the economic performance for all 10 cases under different combinations of uncertainties is shown in TABLE IV. Case 10 has the highest total cost, i.e., $\$ 132000$, which is $21 \%$ higher than that of case 1, since EL-FDIA and GD-FDIA are comprehensively considered with the highest AIL. Case 1 has the lowest economic result since the deterministic model is applied. In addition to the only consideration of FDIA by case 2 , in case 3 , when renewable uncertainty is further considered, the expected total cost increases by $4 \%$. The total economic cost of case 3 is $\$ 13080$, higher than that in case 4 . The main reasons are: i) case 3 considers all three types of FDIA while case 4 only considers EL-FDIA; ii) case 3 considers renewable uncertainty while case 4 does not, iii) case 3 is implemented under RO, which provides more conservative solutions even in the singlestage framework. For case 4, the second stage of DR-FMS considers corrective actions for the day-ahead operation, which accounts for a small portion of the total cost. The big portion of cost is from the first stage, because generation costs for electricity generators and gas wells are considered. From cases 4 to 10 , two-stage DRO is applied, where different types of 
TABLE IV

ECONOMIC PERFORMANCE FOR CASE 1-10

\begin{tabular}{|c|c|c|c|c|c|c|c|c|c|c|}
\hline Economic result & Case 1 & Case 2 & Case 3 & Case 4 & Case 5 & Case 6 & Case 7 & Case 8 & Case 9 & Case 10 \\
\hline First-stage cost $(\$)$ & 108930 & 120955 & 125810 & 108835 & 113922 & 115465 & 119250 & 120880 & 122140 & 123480 \\
\hline $\begin{array}{l}\text { Expected Second- } \\
\text { stage cost }(\$)\end{array}$ & 0 & 0 & 0 & 3895 & 4132 & 4275 & 5292 & 7862 & 8043 & 8520 \\
\hline Total cost $(\$)$ & 108930 & 120955 & 125810 & 112730 & 118054 & 119740 & 124252 & 128742 & 130183 & 132000 \\
\hline
\end{tabular}

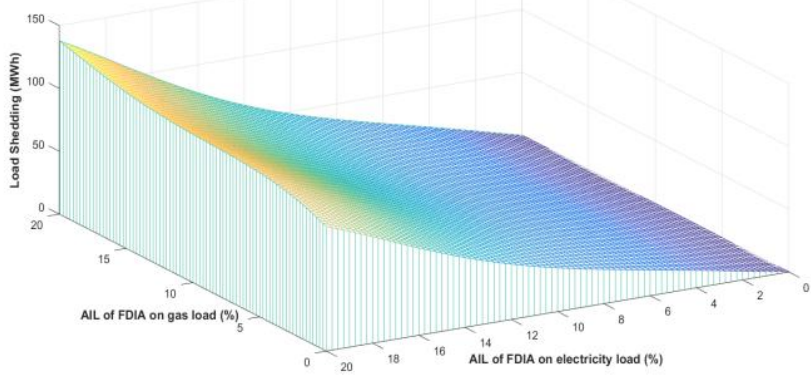

Fig. 4. Electricity load shedding under EL-FDIA and GD-FDIA.

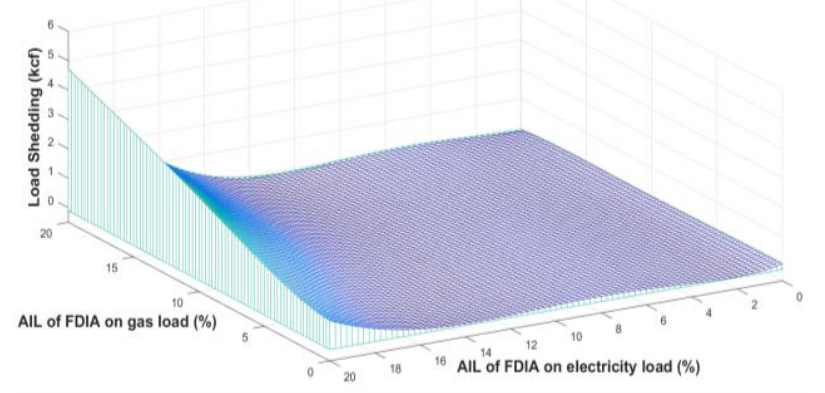

Fig. 5. Gas load shedding under EL-FDIA and GL-FDIA.

FDIA are considered in cases 4-7 and sensitivity analysis is for cases 7-10. Both the first-stage and second-stage expected costs are increasing for cases 4-7 from only considering EL-FDIA to considering all three types of FDIA with renewable uncertainties. It can be found that GL-FDIA has the largest impact on economic performance, i.e., the total cost of case 5 has $4.7 \%$ more cost than case 4 . On the contrary, GD-FDIA has the least impact on economic performance with a $1.4 \%$ rise of total cost from case 5 to case 6 . From case 7 to $10,15 \%$ more AIL causes an increase of total cost from $\$ 124252$ to $\$ 132000$. It should be noted that although EL-FDIA does not increase the overall load increase since some loads are increasing while the rest are decreasing, FDIA aims at attacking critical loads for causing economic losses. Accordingly, under these three types of FDIA with high risks, DR-FMS is more suitable for risk assessment and mitigation considering the worst-distribution.

\section{B. Studies on Load Shedding}

To maintain the feasibility of optimization and system balance under FDIA and renewable uncertainty, it is necessary to implement load shedding. The electricity load shedding (ELS) and gas load shedding (GLS) for 24 hours under FDIA are given in Fig. 4 and 5. In Fig. 4, ELS is up to 140MWh when EL-FDIA and GL-FDIA are both 20\%. ELS is not sensitive to increase when only increasing GL-FDIA level, but sensitive when increasing EL-FDIA. The reason is that the scale of electricity load is much larger than the gas load. Therefore, the GL-FDIA

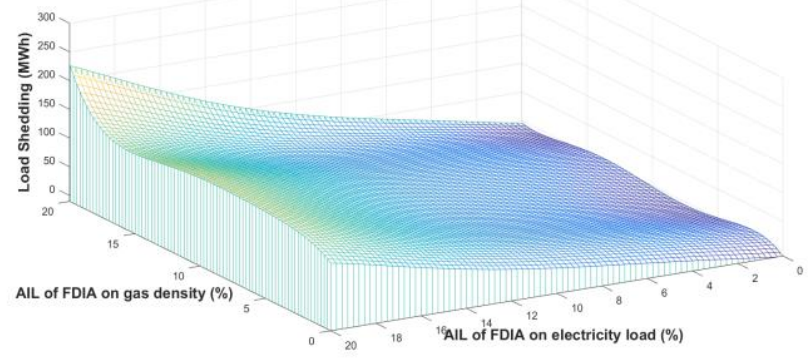

Fig. 6. Electricity load shedding under EL-FDIA and GD-FDIA.

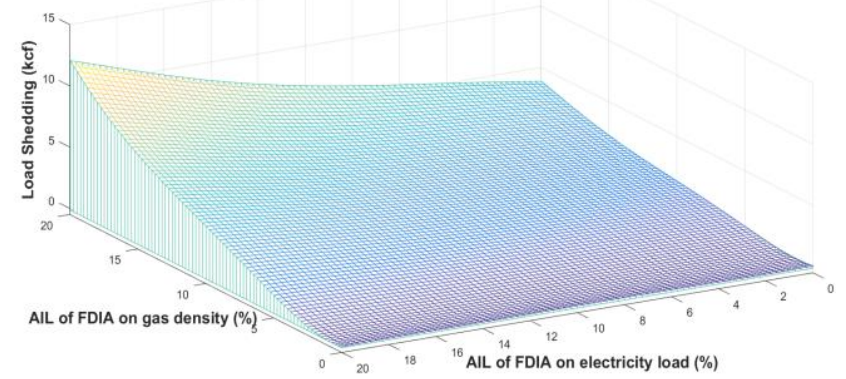

Fig. 7. Gas load shedding under EL-FDIA and GD-FDIA.

TABLE V FCR FOR CASES 4-10

\begin{tabular}{cccc}
\hline \hline FCR & Line 1-2 & Line 6-7 & Line 27-28 \\
\hline Case 4 & $56 \%$ & $56 \%$ & $45 \%$ \\
Case 5 & $57 \%$ & $69 \%$ & $50 \%$ \\
Case 6 & $59 \%$ & $78 \%$ & $54 \%$ \\
Case 7 & $76 \%$ & $87 \%$ & $62 \%$ \\
Case 8 & $89 \%$ & $93 \%$ & $65 \%$ \\
Case 9 & $100 \%$ & $95 \%$ & $80 \%$ \\
Case 10 & $100 \%$ & $96 \%$ & $83 \%$ \\
\hline \hline FCR & Pipeline & Pipeline & Pipeline \\
& N4-N3 & N6-N5 & N1-2 \\
\hline Case 4 & $53 \%$ & $63 \%$ & $64 \%$ \\
Case 5 & $66 \%$ & $65 \%$ & $65 \%$ \\
Case 6 & $77 \%$ & $66 \%$ & $67 \%$ \\
Case 7 & $86 \%$ & $66 \%$ & $90 \%$ \\
Case 8 & $97 \%$ & $68 \%$ & $93 \%$ \\
Case 9 & $100 \%$ & $68 \%$ & $97 \%$ \\
Case 10 & $100 \%$ & $69 \%$ & $100 \%$ \\
\hline \hline
\end{tabular}

has a minor effect on ELS. In Fig. 5, GLS reaches up to $4.7 \mathrm{kcf}$ when FDIA is at the maximum level. GLS increases smoothly when AIL of EL-FDIA is under $17 \%$ while increases significantly when it is over $17 \%$.

The ELS and GLS under EL-FDIA and GD-FDIA are shown in Fig. 6 and Fig. 7. Compared with GL-FDIA in Fig. 4, 84 MWh more ELS is made when considering the GD-FDIA. Since the wrong gas density can directly influence the gas flow. When there is no EL-FDIA, ELS caused by GD-FDIA can still reach up to $3 \mathrm{MWh}$. In Fig. 7, GLS reaches $13 \mathrm{kcf}$ at the maximum AIL compared with the $4.7 \mathrm{kcf}$ in Fig. 5, which again 
TABLE VI

EL-FDIA ON GAS LOAD SHEDDING

\begin{tabular}{|c|c|c|c|c|c|c|c|c|c|c|c|}
\hline AIL & $0 \%$ & $2 \%$ & $4 \%$ & $6 \%$ & $8 \%$ & $10 \%$ & $12 \%$ & $14 \%$ & $16 \%$ & $18 \%$ & $20 \%$ \\
\hline GLS (kcf) & 1.46 & 1.67 & 1.88 & 2.09 & 2.30 & 2.53 & 2.84 & 3.19 & 3.62 & 4.08 & 4.54 \\
\hline \multicolumn{12}{|c|}{$\begin{array}{c}\text { TABLE VII } \\
\text { GL-FDIA ON ELECTRICITY LOAD SHEDDING } \\
\end{array}$} \\
\hline AIL & $0 \%$ & $2 \%$ & $4 \%$ & $6 \%$ & $8 \%$ & $10 \%$ & $12 \%$ & $14 \%$ & $16 \%$ & $18 \%$ & $20 \%$ \\
\hline ELS (MWh) & 34.3 & 36.8 & 39.8 & 39.9 & 41.1 & 41.4 & 42.3 & 42.9 & 43.7 & 48.0 & 48.53 \\
\hline \multicolumn{12}{|c|}{$\begin{array}{c}\text { TABLE VIII } \\
\text { GD-FDIA ON ELECTRICITY LOAD SHEDDING }\end{array}$} \\
\hline AIL & $0 \%$ & $2 \%$ & $4 \%$ & $6 \%$ & $8 \%$ & $10 \%$ & $12 \%$ & $14 \%$ & $16 \%$ & $18 \%$ & $20 \%$ \\
\hline ELS (MWh) & 34.5 & 48.7 & 48.7 & 48.7 & 48.7 & 48.7 & 48.7 & 48.7 & 50.0 & 78.0 & 111.7 \\
\hline
\end{tabular}

proves the significant impact of gas density on GLS. EL-FDIA and GL-FDIA show the similar impact on GLS, i.e., GLS increases by 10kcf when fixing GL-FDIA and increasing ELFDIA while GLS increases by $13 \mathrm{kcf}$ when fixing EL-FDIA and only increasing GL-FDIA. Since as observed from Fig. 4, the scale magnitude of electricity load is much larger than gas load, which largely influences on both ELS and GLS.

As observed from Figs. 4 and 6, the impact of GL-FDIA and GD-FDIA on ELS is minor when EL-FDIA is $0 \%$. It shows that when one type of FDIA is manipulated, the impact on ELS is minor. However, when multiple types of FDIA are conducted in, the impact on ELS could be large. In Fig. 4, with the AIL increase of both GL-FDIA and EL-FDIA, the ELS is massive, which is 99MWh when the AIL of GL-FDIA is $0 \%$ and $139 \mathrm{MWh}$ when AIL of GL-FDIA is $20 \%$. The EL-FDIA is fixed but there is a 40MWh increase of GLS. In Fig. 7, GLS under EL-FDIA and GD-FDIA is given. When GD-FDIA is $0 \%$, EL-FDIA has a low impact on GLS even when the AIL is $20 \%$. However, when AIL of GD-FDIA is at 20\%, GLS ranges from $4.7 \mathrm{kcf}$ to $13 \mathrm{kcf}$, and when AIL of GD-FDIA is at $20 \%$, GLS ranges from $4.7 \mathrm{kcf}$ to $13 \mathrm{kcf}$. This indicates that the security interdependency between electricity and gas systems is minor when attackers only conduct one type of FDIA, but when multiple types of FDIA is attacking the IEGS, it will lead to massive load shedding.

\section{Studies on Flow-Capacity Ratio}

To study the FDIA impact on power and gas flow, in TABLE $\mathrm{V}$, flow-capacity ratio (FCR) for three power lines and three gas pipelines at the peak load time period are studied, which is defined as the percentage of flow divided by the line capacity. The FCR of line N1-N2 and pipeline N4- N3 and N1-2 all reach $100 \%$ when maximum $A I L$ is considered since these three lines play vital parts for interconnecting buses and transmitting flow. From case 4 to 7, there is a general increase for FCR of power lines and gas pipelines since types of FDIA are gradually incorporated. From cases 7 to 10, the FCR still monotonically increases when $A I L$ is increasing from $5 \%$ to $20 \%$. It should be noted that line 1-2 and line 27-28 are more sensitive to increase of $A I L$ with a $24 \%$ and $21 \%$ increase respectively. Compared to pipeline N4-N3 which is prone to overloading, the FCR of pipeline N6-N5 ranges only reaches 69\%, indicating that gas flow is mainly sourced from the gas well connected to node 4.

\section{Discussion on System Interdependency under FDIA}

The interdependency between electricity and gas systems worsen the system security, i.e., the FDIA on electricity system has adverse impact on gas system and vice versa. TABLE VIVIII present the impact of EL-FDIA on GLS, GL-FDIA on ELS and GD-FDIA on ELS, respectively. The EL-FDIA has minor impact on gas system compared with GL-FDIA on gas system and GL-FDIA or GD-FDIA have also minor impact on electricity system compared with EL-FDIA on electricity system. In order to observe a more obvious result, TABLE VI is under the fixed $10 \%$ of GD-FDIA and TABLE VII and VIII are under the fixed $10 \%$ of EL-FDIA.

A smooth increase of GLS is shown in TABLE VI, i.e., the increase of GLS with the $20 \%$ increase of EL-FDIA is only $3 \mathrm{kcf}$. The GLS accounts for $1 \%$ when EL-FDIA is at $20 \%$. The potential reasons for this result are i) the electricity system is resilient enough based on multiple electricity generators and renewable generators which does not require massive supply from gas system and ii) the overall operation cost and load shedding of IEGS will increase significantly provided that gas system provides more supply to electricity system while implements more GLS. In TABLE VII, GL-FDIA causes 48.5MWh ELS when $A I L$ is at $20 \%$. The result is on the contrary of TABLE VI since GL-FDIA causes gas wells supply more on gas system itself, which can be also viewed from Fig. 5. The increase of GL-FDIA does not result in significant increase of GLS, which indicates that there is far less gas to power flow. In TABLE VIII, GD-FDIA shows a more severe impact on ELS. The ELS when GD-FDIA is $0 \%$ is only 34.5MWh while it increases dramatically and reaches 111.7 when GD-FDIA is $20 \%$. The reason is that GD-FDIA directly increases the gas density, which accordingly limits the gas flow and the gas turbine production is restricted.

\section{E. Comparison with RO Based Mitigation Scheme}

The comparison made between DR-FMS and R-FMS is in Figs. 8 and 9. In general, the (flow-capacity ratio) FCR from RFMS is higher than that from DR-FMS for all power lines and gas pipelines. In Fig. 8, the FCR in pipeline N1-2 has the highest level. The FCR solved by DR-FMS is $90 \%$ and $98 \%$ by R-FMS. Pipeline N4-N3 has the second-highest FCR and the difference between DR-FMS and R-FMS is 9\%. Fig. 9 depicts the FCR at the lowest load period, which shows lower FCR for all power lines and gas pipelines. The largest FCR difference from DR-FMS and R-FMS is for line 27-28, which is $13 \%$. For line 6-7, the FCR difference modelled by two schemes is the lowest for both peak and lowest load period. To summarize, DR-FMS not only provide more economic operation scheme 


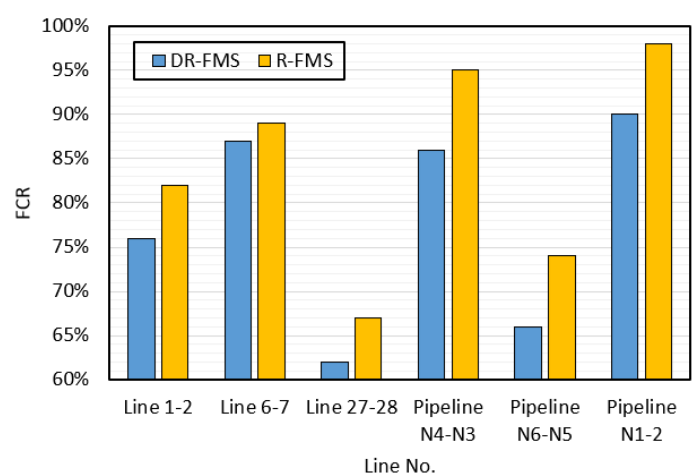

Fig. 8. FCR for power lines and gas pipelines at peak load period.

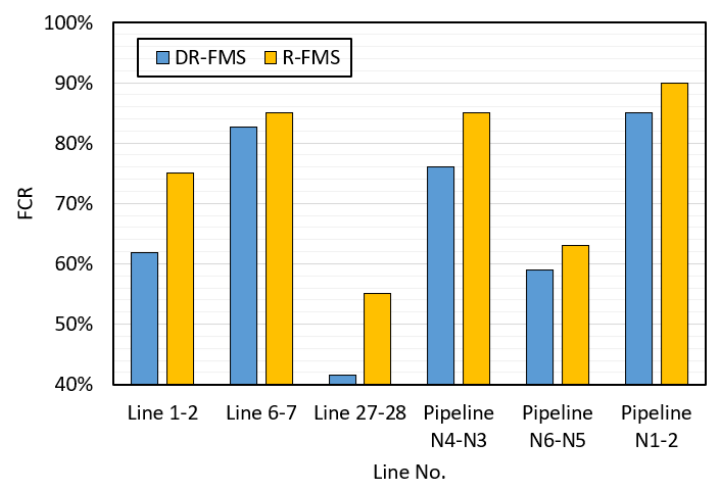

Fig. 9. FCR for power lines and gas pipelines at lowest load period.

but yields lower FCR since the FDIA is considered in a moderate robust manner.

\section{CONCLUSION}

A risk mitigation scheme for IEGS against FDIA is proposed in this paper with a two-stage DRO model. A tractable SDP formulation is built for the original DR-FMS, which is solved by CGA in an iterative manner. Through the extensive case studies, the key findings are listed below:

- Considering all three types of FDIA, i.e., EL-FDIA, GLFDIA and GD-FDIA, leads to higher economic results and more load shedding than considering two types or one type of FDIA. Load shedding is more sensitive to EL-FDIA than GD-FDIA or GL-FDIA.

- DRO provides less-conservative results than RO in terms of economic performance and load shedding.

- Renewable generation uncertainty is necessary to consider, which leads to $3.7 \%$ more operation cost.

The proposed DR-FMS ensures the economic performance of IEGS by providing a two-stage risk mitigation scheme via implementing efficient load shedding under FDIA and renewable uncertainty. The beneficiaries of this work inlcude: network operators can have powerful operation models, end customers will enjoy better supply security, and renewable can penetrate to the maximum level without much curtailment.

\section{REFERENCES}

[1]A. Mahmood, N. Javaid, and S. Razzaq, "A review of wireless communications for smart grid," Renewable and sustainable energy reviews, vol. 41, pp. 248-260, 2015.

[2] G. Liang, J. Zhao, F. Luo, S. R. Weller, and Z. Y. Dong, "A Review of False Data Injection Attacks Against Modern Power Systems," IEEE Transactions on Smart Grid, vol. 8, pp. 1630-1638, 2017.
[3] G. Liang, S. R. Weller, J. Zhao, F. Luo, and Z. Y. Dong, "The 2015 ukraine blackout: Implications for false data injection attacks," IEEE Transactions on Power Systems, vol. 32, pp. 3317-3318, 2016.

[4]L. Che, X. Liu, Z. Li, and Y. Wen, "False Data Injection Attacks Induced Sequential Outages in Power Systems," IEEE Transactions on Power Systems, vol. 34, pp. 1513-1523, 2019.

[5]A. Tajer, "False Data Injection Attacks in Electricity Markets by Limited Adversaries: Stochastic Robustness," IEEE Transactions on Smart Grid, vol. 10, pp. 128-138, 2019.

[6]R. Deng, P. Zhuang, and H. Liang, "False Data Injection Attacks Against State Estimation in Power Distribution Systems," IEEE Transactions on Smart Grid, vol. 10, pp. 2871-2881, 2019.

[7]A. Ashok, M. Govindarasu, and V. Ajjarapu, "Online Detection of Stealthy False Data Injection Attacks in Power System State Estimation," IEEE Transactions on Smart Grid, vol. 9, pp. 1636-1646, 2018.

[8] X. Wang, X. Luo, Y. Zhang, and X. Guan, "Detection and Isolation of False Data Injection Attacks in Smart Grids via Nonlinear Interval Observer," IEEE Internet of Things Journal, pp. 1-1, 2019.

[9]Y. Yuan, Z. Li, and K. Ren, "Modeling Load Redistribution Attacks in Power Systems," IEEE Transactions on Smart Grid, vol. 2, pp. 382-390, 2011. [10] Y. Yuan, Z. Li, and K. Ren, "Quantitative Analysis of Load Redistribution Attacks in Power Systems," IEEE Transactions on Parallel and Distributed Systems, vol. 23, pp. 1731-1738, 2012.

[11] L. Che, X. Liu, and Z. Li, "Mitigating False Data Attacks Induced Overloads Using a Corrective Dispatch Scheme," IEEE Transactions on Smart Grid, vol. 10, pp. 3081-3091, 2019.

[12] H. Shayan and T. Amraee, "Network Constrained Unit Commitment Under Cyber Attacks Driven Overloads," IEEE Transactions on Smart Grid, pp. 1-1, 2019.

[13] Z. Li, W. Wu, M. Shahidehpour, J. Wang, and B. Zhang, "Combined Heat and Power Dispatch Considering Pipeline Energy Storage of District Heating Network," IEEE Transactions on Sustainable Energy, vol. 7, pp. 1222, 2016.

[14] Y. Zhou, W. Hu, Y. Min, and Y. Dai, "Integrated Power and Heat Dispatch Considering Available Reserve of Combined Heat and Power Units," IEEE Transactions on Sustainable Energy, vol. 10, pp. 1300-1310, 2019.

[15] C. Gu, C. Tang, Y. Xiang, and D. Xie, "Power-to-gas management using robust optimisation in integrated energy systems," Applied Energy, vol. 236, pp. 681-689, 2019/02/15/ 2019.

[16] J. Yang, N. Zhang, Y. Cheng, C. Kang, and Q. Xia, "Modeling the Operation Mechanism of Combined P2G and Gas-Fired Plant With $\mathrm{CO} 2$ Recycling," IEEE Transactions on Smart Grid, vol. 10, pp. 1111-1121, 2019. [17] S. Massucco, A. Pitto, and F. Silvestro, "A Gas Turbine Model for Studies on Distributed Generation Penetration Into Distribution Networks," IEEE Transactions on Power Systems, vol. 26, pp. 992-999, 2011.

[18] R. Rigo-Mariani, C. Zhang, A. Romagnoli, M. Kraft, K. V. Ling, and J. Maciejowski, "A Combined Cycle Gas Turbine Model for Heat and Power Dispatch Subject to Grid Constraints," IEEE Transactions on Sustainable Energy, vol. 11, pp. 448-456, 2020.

[19] A. Nikoobakht, J. Aghaei, M. Shafie-khah, and J. P. S. Catalão, "Continuous-Time Co-Operation of Integrated Electricity and Natural Gas Systems with Responsive Demands Under Wind Power Generation Uncertainty," IEEE Transactions on Smart Grid, pp. 1-1, 2020.

[20] Y. Li, Y. Zou, Y. Tan, Y. Cao, X. Liu, M. Shahidehpour, et al., "Optimal Stochastic Operation of Integrated Low-Carbon Electric Power, Natural Gas, and Heat Delivery System," IEEE Transactions on Sustainable Energy, vol. 9, pp. 273-283, 2018.

[21] Y. He, M. Shahidehpour, Z. Li, C. Guo, and B. Zhu, "Robust Constrained Operation of Integrated Electricity-Natural Gas System Considering Distributed Natural Gas Storage," IEEE Transactions on Sustainable Energy, vol. 9, pp. 1061-1071, 2018.

[22] C. He, C. Dai, L. Wu, and T. Liu, "Robust Network Hardening Strategy for Enhancing Resilience of Integrated Electricity and Natural Gas Distribution Systems Against Natural Disasters," IEEE Transactions on Power Systems, vol. 33, pp. 5787-5798, 2018.

[23] Y. Li, Z. Li, F. Wen, and M. Shahidehpour, "Minimax-Regret Robust Co-Optimization for Enhancing the Resilience of Integrated Power Distribution and Natural Gas Systems," IEEE Transactions on Sustainable Energy, vol. 11, pp. 61-71, 2020.

[24] X. Lu, K. W. Chan, S. Xia, B. Zhou, and X. Luo, "Security-Constrained Multiperiod Economic Dispatch With Renewable Energy Utilizing Distributionally Robust Optimization," IEEE Transactions on Sustainable Energy, vol. 10, pp. 768-779, 2019. 
[25] P. Zhao, H. Wu, C. Gu, and I. H. Gil, "Optimal Home Energy Management under Hybrid PV-Storage Uncertainty: A Distributionally Robust Chance-Constrained Approach," IET Renewable Power Generation, p. 9, 2019.

[26] C. Wang, R. Gao, W. Wei, M. Shafie-khah, T. Bi, and J. P. S. Catalão, "Risk-Based Distributionally Robust Optimal Gas-Power Flow With Wasserstein Distance," IEEE Transactions on Power Systems, vol. 34, pp. 2190-2204, 2019.

[27] L. Wu, C. He, X. Zhang, and T. Liu, "Distributionally Robust Scheduling of Integrated Gas-Electricity Systems with Demand Response," IEEE Transactions on Power Systems, pp. 1-1, 2019.

[28] P. Zhao, C. Gu, D. Huo, Y. Shen, and I. Hernando-Gil, "Two-Stage Distributionally Robust Optimization for Energy Hub Systems," IEEE Transactions on Industrial Informatics, vol. 16, pp. 3460-3469, 2020.

[29] T. Ding, C. Li, Y. Yang, J. Jiang, Z. Bie, and F. Blaabjerg, "A Two-Stage Robust Optimization for Centralized-Optimal Dispatch of Photovoltaic Inverters in Active Distribution Networks," IEEE Transactions on Sustainable Energy, vol. 8, pp. 744-754, 2017.

[30] H. Gao, J. Liu, and L. Wang, "Robust Coordinated Optimization of Active and Reactive Power in Active Distribution Systems," IEEE Transactions on Smart Grid, vol. 9, pp. 4436-4447, 2018.

[31] M. Qadrdan, J. Wu, N. Jenkins, and J. Ekanayake, "Operating Strategies for a GB Integrated Gas and Electricity Network Considering the Uncertainty in Wind Power Forecasts," IEEE Transactions on Sustainable Energy, vol. 5, pp. 128-138, 2014.

[32] B. Odetayo, J. MacCormack, W. D. Rosehart, and H. Zareipour, "A sequential planning approach for Distributed generation and natural gas networks," Energy, vol. 127, pp. 428-437, 2017/05/15/ 2017.

[33] R. Mageshvaran and T. Jayabarathi, "Steady state load shedding to mitigate blackout in power systems using an improved harmony search algorithm," Ain Shams Engineering Journal, vol. 6, pp. 819-834, 2015/09/01/ 2015.

[34] C. M. Correa-Posada and P. Sánchez-Martín, "Integrated Power and Natural Gas Model for Energy Adequacy in Short-Term Operation," IEEE Transactions on Power Systems, vol. 30, pp. 3347-3355, 2015.

[35] E. Delage and Y. Ye, "Distributionally robust optimization under moment uncertainty with application to data-driven problems," Operations research, vol. 58, pp. 595-612, 2010.

[36] Y. Chen, W. Wei, F. Liu, and S. Mei, "Distributionally robust hydrothermal-wind economic dispatch," Applied Energy, vol. 173, pp. 511-519, 2016/07/01/ 2016.

[37] A. S. Musleh, G. Chen, and Z. Y. Dong, "A Survey on the Detection Algorithms for False Data Injection Attacks in Smart Grids," IEEE Transactions on Smart Grid, pp. 1-1, 2019.

[38] I. I. o. T. Electrical and Computer Engineering Department. Index of /data. Available: motor.ece.iit.edu/data/

[39] R. J. Kuo and C. C. Huang, "Application of particle swarm optimization algorithm for solving bi-level linear programming problem," Computers \& Mathematics with Applications, vol. 58, pp. 678-685, 2009/08/01/ 2009.

[40] S. Sengupta, S. Basak, and R. A. Peters, "Particle Swarm Optimization: A survey of historical and recent developments with hybridization perspectives," Machine Learning and Knowledge Extraction, vol. 1, pp. 157191, 2019.

[41] R. J. Kuo and Y. S. Han, "A hybrid of genetic algorithm and particle swarm optimization for solving bi-level linear programming problem - A case study on supply chain model," Applied Mathematical Modelling, vol. 35, pp. 3905-3917, 2011/08/01/ 2011.

[42] D. E. Kvasov and M. S. Mukhametzhanov, "Metaheuristic vs. deterministic global optimization algorithms: The univariate case," Applied Mathematics and Computation, vol. 318, pp. 245-259, 2018/02/01/ 2018.

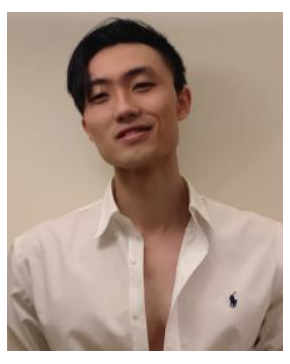

Pengfei Zhao (S'18) was born in Beijing, China. He received the double B.Eng. degree from the University of Bath, U.K., and North China Electric Power University, China, in 2017. He is currently pursuing the Ph.D. degree with the Department of Electronic and Electrical Engineering, University of Bath, U.K. He was a visiting Ph.D. student at Tsinghua University, Beijing, China in 2019. His research interests include the operation and planning of integrated energy systems considering inherent uncertainties.

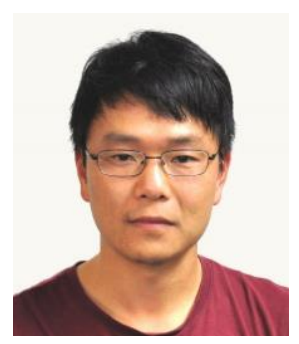

Chenghong Gu (M'14) was born in Anhui province, China. He received the Master's degree from the Shanghai Jiao Tong University, Shanghai, China, in 2007 in electrical engineering. He received the Ph.D. degree from the University of Bath, U.K. He is currently a Lecturer and EPSRC Fellow with the Department of Electronic and Electrical Engineering, University of Bath. His major research interest is in multi-vector energy system, smart grid, and power economics.

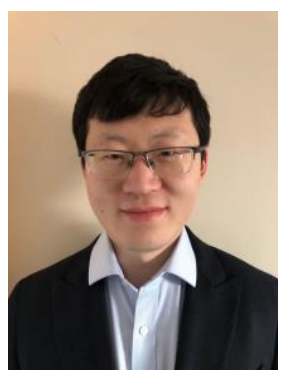

Da Huo was born in Inner Mongolia, China. He received the B.Eng. degrees in electrical and electronic engineering from the University of Bath, U.K., and in electrical power engineering from North China Electric Power University, Baoding, China, in 2014. He received the Ph.D. degree from the University of Bath, U.K, in 2018. He is currently a research associate with the School of Engineering, Newcastle University. His main research interests are multi-carrier energy system and smart grid. 